\title{
Investigating the psychometric properties of the Rosenberg Self-Esteem Scale (RSES) for South African residents of Greater Pretoria
}

\author{
Margaret S. Westaway, PhD, ${ }^{1,2, *}$ \\ Esmè R. Jordaan, MSc, ${ }^{3}$ \\ Jennifer Tsai, MPH ${ }^{4}$
}

\footnotetext{
${ }^{1}$ Health and Development Research Unit, Medical Research Council, Pretoria, South Africa

${ }^{2}$ School of Health Systems and Public Health, Faculty of Health Sciences, University of Pretoria, Pretoria, South Africa

${ }^{3}$ Biostatistics Unit, Medical Research Council, Cape Town, South Africa

${ }^{4}$ Departments of Preventive Medicine and Psychology, University of Southern California, Los Angeles, CA, USA
}

*Corresponding author. Address: Health and Development Research Unit, Medical Research Council, Private Bag X385, Pretoria 0001

Tel.: +27 12339 8537; fax: +27 123398594 .

E-mail addresses: 27824472294@ vodamail.co.za; margaretw@ vodamail.co.za; Margaret.Westaway@ mrc.ac.za

\begin{abstract}
Interviewers administered the Rosenberg Self-Esteem Scale (RSES) to five groups of Black, White, Indian and Mixed Race adult residents of Greater Pretoria. The results demonstrated that the RSES was psychometrically sound for the five groups. The minimal effects of sociodemographic characteristics on global self-esteem, self-competence (SC), and self-liking (SL) showed that the RSES and its two dimensions are suitable for use in this setting. All five groups scored above the theoretical midpoint of the RSES, indicating that generally positive self-evaluations appear to be universal. The relationships between positively and negatively
\end{abstract}


worded items, SC, and SL attested to the following: internal structure reliability, congruence between positive and negative items, no negative biases in response, and concordance between SC and SL dimensions. In this South African setting, individualism-collectivism did not appear to play a role in self-evaluation. The significant differences between informal settlement residents and the other four groups on global self-esteem, positively and negatively worded items, and SC and SL was possibly due to physiological needs taking precedence over higher order needs.

Keywords : Rosenberg Self-Esteem scale, reliability, item validity, principal components analysis, greater Pretoria residents

\section{Introduction}

Rosenberg (1965) defined self-esteem as "a positive or negative attitude toward a particular object, namely, the self' (p. 30). Individuals who take a positive attitude towards themselves do not necessarily feel superior to others or accept themselves unconditionally, but they do respect themselves and consider themselves worthy. Furthermore, they recognize their limitations and have expectations concerning their growth and improvement (Rosenberg, 1965). Rosenberg developed a 10-item scale to measure the self-acceptance aspect of selfesteem, and validated it with a sample of 5,024 adolescents from New York. This scale contains an equal number of positively and negatively worded items, originally conceptualized by Rosenberg (1965) as a single factor structure, with scores ranging along a continuum from low to high self-esteem.

There has been considerable debate on the dual versus single factor structure of the Rosenberg Self-Esteem Scale (Bornman, 1999; Carmines \& Zeller, 1979; Francis \& James, 1996; Greenberger, Chen, Dmitrieva \& Farruggia, 2003; Hatcher \& Hall, 2009; Pullmann \& Allik, 2000). Carmines and Zeller (1979) reported a two-factor positive and negative self- 
esteem structure. However, by correlating the positive and negative self-esteem factors with theoretically relevant variables, they were able to show that there was "a single theoretical dimension of self-esteem that is contaminated by a method artifact, response set" (p. 69). An alternative analysis for internal structure reliability is to correlate the sum of the positively and negatively worded items (Schmitt \& Allik, 2005), establishing congruence between positive and negative items.

One problematic issue for the RSES is the effect of individualism-collectivism on selfesteem (Triandis, 1995). Individualistic definitions of the self-concept as a discrete, independent, and abstract self place a higher priority on the person's own goals, whereas collectivism refers to interdependence (fitting in, belonging, and becoming part of relevant social relationships) and a concrete self-concept where the group's goals receive a higher priority than the individual's goals (Triandis, 1995). In a South African study, with university students, African-language students tended towards interdependence with a concrete selfconcept, than English-language students, providing some support for the relationship between collectivism and self-esteem (Eaton \& Louw, 2000). In contrast, Diener and Diener (1995) found no relationship between self-satisfaction, based on a single item of self-esteem and individualism in their study with college students from 31 countries. Schmitt and Allik (2005) also found a negligible correlation between national RSES scores and Hofstede's Individualism Index (Hofstede, 2001). It is possible that these contrasting findings were based on methodological differences between studies, such as the use of the Twenty Statements Test (20 responses to the question: Who am I?) by Eaton and Louw (2000), a single item measure of self-esteem (Diener \& Diener, 1995), and the 10-item RSES (Schmitt \& Allik, 2005).

In recent years, Tafarodi and his colleagues (Tafarodi, Lang \& Smith, 1999; Tafarodi \& Milne, 2002; Tafarodi \& Swann, 1995) have divided the RSES into two equal halves: self- 
competence (SC) and self-liking (SL). Self-competence (SC) refers to the evaluation of oneself as capable, effective, and confident, whereas self-liking (SL) is socially dependent and relates to an affective judgment of oneself as socially relevant.

Tafarodi, Lang, and Smith (1999) reported that individualistic cultures, such as the United Kingdom (UK), scored higher on the SC dimension of self-esteem than Malaysia (collectivistic culture) and vice versa for the SL dimension of self-esteem. Schmitt and Allik (2005) compared the UK and Malaysia on SC and SL but did not replicate this finding. Although there were no differences between the two groups on SC, the UK scored significantly higher on SL than Malaysia. Controlling for gender and SL, Schmitt and Allik (2005) found that the five most individualistic cultures (Australia, Brazil, the Netherlands, the UK, and US) scored significantly higher on the SC dimension than the five most collectivistic cultures (Bangladesh, Botswana, Indonesia, Peru and South Korea), providing some evidence of the relationship between individualism-collectivism and self-competence (SC). Although African cultures are assumed to be collectivistic (Triandis, 1995), it is highly likely that acculturation, rapid urbanization, and globalization tend to restrict collectivism to rural rather than urban areas (Naidoo \& Mahabeer, 2006).

An alternative explanation for the differences between and within countries on global self-esteem, and the self-competence (SC) and self-liking (SL) dimensions is that of Maslow's (1954) "hierarchy of needs". He posited that certain deficiencies that must be satisfied before one may progress to the next highest level largely determine behavior. There are four need levels driven by deficiency: physiological, safety and security, belongingness and love, and esteem needs (Maslow, 1954). Although he believed that human needs formed a hierarchy, he added the caveat that it was not necessarily a rigid one. For example, in some individuals, the need for self-esteem appeared to over-ride that of belongingness and love. In others, creativity (usually associated with self-actualization) flourished in spite of deficits in 
the fulfillment of lower-level needs. However, gratification of basic needs is essential for human functioning and survival, and tends to take precedence over self-esteem needs in disadvantaged communities (Veenhoven, 1996; Westaway, 2006).

In summary, the RSES is a well-validated measure of the construct global self-esteem. Firstly, the RSES has a predominantly one-factor structure, and positive self-esteem appears to be culturally universal, since scores are generally above the theoretical mid-point. Secondly, global self-esteem is higher in Western than Eastern countries and individualistic cultures tend to score higher on the self-competence (SC) dimension than collectivistic cultures (Rosenberg, 1965; Schmitt \& Allik, 2005; Sinclair et al., 2010). Thirdly, an alternative explanation for differences between and within countries on global self-esteem, self-competence, and self-liking is Maslow’s (1954) hierarchy of needs.

\section{Rationale for the Study}

Although the RSES is a well-validated measure, South Africa provides a unique multicultural, multi-racial setting for extending an already robust RSES literature since findings from the current study can complement and/or conflict with previous research (i.e., Schmitt \& Allik, 2005; Sinclair et al., 2010). In particular, the use of community samples for the current study addressed Butler and Gasson's (2006) critique on limited samples (college students and/or convenience sampling). A multi-method approach was taken for data analysis, addressing concerns about the use of factor analysis alone to evaluate structure (Sinclair et al., 2010), and the focus on self-competence (SC) and self-liking (SL) advances our knowledge of the relationship between individualism-collectivism and these dimensions.

The objectives of the study were: (1) Evaluate scaling assumptions (equivalent item variance, item convergent and discriminant validity, internal consistency reliability, floor and ceiling effects, and component structure) underlying the RSES for five groups (formerly Black only formal township and informal settlement residents, and formerly White, Indian, 
and Mixed Race only suburbanites of Greater Pretoria). (2) Ascertain the combined effects of socio-demographic characteristics (age, gender, schooling, marital status, and employment status) on the RSES, self-competence (SC) and self-liking (SL). (3) Investigate group effects on the RSES, SC, SL, and positively and negatively worded items. (4) Investigate whether Maslow's (1954) hierarchy of needs is an alternative explanation to the individualismcollectivism debate on self-competence and self-liking. (5) Compare findings for the five groups with those from Botswana and Zimbabwe (Schmitt \& Allik, 2005).

\section{Method}

\section{Sampling and participants}

Due to the high crime rate, Indian, White, and Mixed Race residents have barricaded their homes, many with guns and fierce dogs for additional protection. In our experience of fieldwork, it is dangerous for fieldworkers to enter these areas without making prior contact (Westaway, 2007). Therefore, for the Indian, White, and Mixed Race samples, sampling consisted of social clubs, senior citizens clubs, retirement centers, and complexes. For these three groups, the interviewers made appointments to administer the measures in the prospective respondents' own home or at these facilities. This sampling procedure was essential as Indian, White, and Mixed Race South Africans show considerable reluctance to participate in research activities.

With the exception of Church groups, there are minimal social amenities in Pretoria's Black formal townships and informal settlements. Therefore, a simple random sample drew 450 stand numbers from the Black well-established formal township, and 500 stand numbers from the Black informal settlement. There were only 19 refusals in the formal township and 26 in the informal settlement, all due to time constraints, indicating that Black South Africans are very willing to participate in research activities. 
Participants were 1158 Black, White, Indian, and Mixed Race adult residents (aged 18 and older) from five historically distinct areas of Greater Pretoria (formerly Black only formal township and informal settlement, formerly White only suburb, formerly Indian only suburb, and formerly Mixed Race only suburb). There were 400 formal township and 448 informal settlement residents, 107 White residents, 101 Indian residents, and 102 Mixed Race residents. There were more women than men and a wide distribution of age ( $M=57.3$ years, $S D=19.1$, range $=18-93$ ). The major languages were Sotho and Zulu for Black respondents. Afrikaans was the major language for White respondents and Mixed Race respondents, and English for Indian respondents. Years of education ranged between none and $12(M=7.7, S D$ $=3.4$ ). However, $39 \%$ of the sample had seven or less years of formal schooling, indicative of functional illiteracy, and a lack of educational opportunities for Black, Indian, and Mixed Race South Africans. Due to the wide age range, there were approximately equal numbers of married (37\%) and widowed (31\%) respondents, but only $20 \%$ were employed, predominantly informal settlement residents. The major source of income for formal township residents, White, Indian, and Mixed Race respondents, was a non-contributory government or a contributory private pension.

There were no differences between the five groups on gender $(p=0.41)$. As expected, White respondents were significantly older than Black, Indian, and Mixed Race respondents ( $p<0.001$ ), since $33 \%$ of White, $20 \%$ of Indian, $14 \%$ of Mixed Race, and $12 \%$ of Black South Africans are aged 50 and older (Lehohla, 2006). White South Africans comprise a more rapidly aging population than the other population groups. Informal settlement residents were significantly younger than formal township, White, Indian, and Mixed Race residents ( $p$ $<0.001)$. They were also more likely to be working, had received more education than formal township residents and Mixed Race residents, and were less likely to be widowed $(p<0.001)$ than the other four groups. 


\section{Materials}

The RSES. The RSES consists of 10 items rated from 1 (strongly disagree) to 4 (strongly agree), with 5 items reverse scored (Q3, Q5, Q8, Q9 and Q10). Scoring consisted of a summation of the 10 items for an overall self-esteem score, with a range of 10 to 40 (Schmitt \& Allik, 2005).The current study used the same scoring system of Schmitt and Allik (2005) to allow for comparisons with findings from Botswana and Zimbabwe. Selfcompetence (SC) refers to a summation of the first five items of the RSES and self-liking (SL) a summation of the last five items. The range for SC and SL is 5 to 20 , respectively (Tafarodi \& Milne, 2002).

Self-assessed health. A frequently used single item measure that asks respondents to rate their overall health as poor, fair, good, very good, or excellent assessed health status. Numerous studies report that self-assessed health is not only a reliable and valid measure of a population's health and well-being, but also a strong predictor of morbidity, mortality, and health care utilization (Alexopoulos \& Geitona, 2010; Gilmore, McKee \& Rose, 2002; Idler \& Benjamin, 1997; Meurer, Layde \& Guse, 2001).

\section{Procedure}

Due to the high rates of functional illiteracy and a lack of questionnaire sophistication among Blacks, Indians, Mixed Races, and Whites (Afrikaans-speaking), the RSES, along with selfassessed health status, and socio-demographic characteristics (gender, age, completed years of formal schooling, marital status, and employment status), was administered by 25 multilingual, trained and paid interviewers in the five study sites. The interviewers conducted faceto-face interviews with all respondents in the preferred language of the respondents (Sotho, Zulu, Afrikaans, or English). Black interviewers interviewed Black participants, and White, 
Indian, and Mixed Race interviewers interviewed White, Indian, and Mixed Race respondents, respectively. All interviewers paid particular attention to the consent form, in accordance with the World Association Declaration of Helsinki Ethical Principles for Medical Research involving Human Subjects.

\section{Data analysis}

Descriptive statistics were the first step for data analysis. For each of the five groups, linear regression analyses examined the combined effects of socio-demographic characteristics (age, gender, schooling, marital status, and employment status) on the RSES, and selfcompetence (SC) and self-liking (SL). Pearson product-moment correlation coefficients examined the relationship between positively and negatively worded items of the RSES (internal structure reliability), and the relationship between its two dimensions SC and SL. One Way Analysis of Variance (ANOVA), with Bonferroni adjustments for multiple comparisons, ascertained group effects on the RSES, its two components SC and SL, and positively and negatively worded items.

Psychometric analyses consisted of multi-trait scaling (equivalent item variance, item convergent, and discriminant validity), internal consistency reliability, floor and ceiling effects, and component structure of the RSES. Multi-trait scaling was the first step in evaluating scaling assumptions (Stewart, Hays \& Ware, 1988). This method consists of three steps designed to establish whether items have equivalent variance, item convergent, and discriminant validity. For item convergent validity, corrected item-total correlation coefficients are the relationship between the specific item and a summation of the other items in the scale. The criterion for corrected item-total correlation coefficients was set at $r>0.40$ (Stewart, Hays \& Ware, 1988). For item discriminant validity, the correlation between an item and its hypothesized scale needs to be significantly higher than with other scales 
measuring different concepts (Sinclair et al., 2010). For this analysis, the health rating scale measured a different concept to self-esteem. Steiger's $t$ tests (Steiger, 1980) for dependent correlations evaluated whether the 10 RSES items correlated significantly higher with their own scale than the health rating scale.

Coefficient alpha determined the reliability (internal consistency) of the RSES for each of the five groups (Arias \& de Vos, 1996; Cronbach, 1970; George \& Mallery, 2003; Nunnally, 1978). The intra-class correlation (ICC) for average measures, with a two-way mixed effects model, where people effects are random and measure effects are fixed, determined the lower and upper bound for alpha at the 95\% confidence interval (CI).

Floor and ceiling effects examined the percentage of respondents achieving either the lowest score (floor) or the highest score (ceiling). Where a substantial proportion of respondents score at either the floor or ceiling indicates that the range of the scale is inappropriate, or the items do not adequately assess the construct self-esteem in particular populations (Sinclair et al., 2010, p. 72).

A parallel analysis ascertained extraction of the number of components. This procedure involves extracting eigenvalues from random data sets that parallel the actual data set with regard to the number of cases and variables. The eigenvalues derived from the actual data compared the eigenvalues from the random data (O’Connor, 2000). This procedure is statistically superior to examining eigenvalues-greater-than-one or the scree plots of eigenvalues (O’Connor, 2000). Principal components analysis (PCA) compared findings with those of Schmitt and Allik (2005).

\section{Ethics}

Ethical approval for the study came from the Faculty of Health Sciences Ethical Committee, University of Pretoria, and approval by their Institutional Review Board (IRB) for use of 
human subjects. There were consultations with Greater Pretoria community structures regarding the study. All respondents who were willing to participate in the study gave their written informed consent.

\section{Results}

Psychometric Evaluation of the RSES

Equivalent item variance. For the overall sample, variance ranged between 0.36 and 0.38. Although the range for each of the five groups was higher than this, low variance of responses on the RSES indicated that responses were roughly comparable for all items, meeting Stewart, Hays and Ware's (1988) first criterion of equivalent item means and variance, and provided support for Baumeister and Tice's (1988) meta-trait hypothesis (Table $1)$.

Item convergent validity. For each of the five groups, all 10 corrected item-total correlation coefficients were higher than the criterion of $>0.40$, providing evidence for item convergent validity (Stewart, Hays \& Ware, 1988), Table 1.

Item discriminant validity. All 10 self-esteem items correlated significantly higher ( $p$ $<0.05)$ with their own scale than the health rating scale. Steiger's (1980) $t$ tests ranged between 24.4 and 29.2 for the overall sample, between 11.4 and 14.8 for the informal settlement group, between 13.5 and 19.1 for the formal settlement group, between 6.7 and 13.8 for the Indian group, between 3.3 and 9.3 for the White group, and between 8.2 and 11.7 for the Mixed Race group (Table 1).

Internal consistency reliability. Reliability (internal consistency) coefficients for the RSES were excellent (George \& Mallery, 2003; Nunnally, 1978), with Cronbach's coefficient alpha ranging from 0.93 (Informal settlement: ICC 95\% CI: 0.92-0.94), 0.94 (White respondents: ICC 95\% CI: 0.92-0.96) to 0.97 (Formal township: ICC 95\% CI: 0.97- 
Table I. Multitrait Scaling (Equivalent Item Variance, Item Convergent, and Discriminant Validity), Range of Steiger's t-tests, Internal Consistency Reliability, and Floor and Ceiling Percentages Overall and for the Five Groups.

\begin{tabular}{|c|c|c|c|c|c|c|c|c|}
\hline & $N$ & $\begin{array}{l}\text { Equivalent Item } \\
\text { Variance }^{\mathrm{a}}\end{array}$ & $\begin{array}{c}\text { Item Convergent } \\
\text { Validity }^{\mathrm{b}}\end{array}$ & $\begin{array}{l}\text { Item Discrimi- } \\
\text { nant Validityc }\end{array}$ & $\begin{array}{l}\text { Range Steiger's } \\
\text { t-tests }{ }^{d}\end{array}$ & $\begin{array}{c}\text { Internal Consistency } \\
\text { Reliability }\end{array}$ & $\begin{array}{l}\% \\
\text { Floor }\end{array}$ & $\begin{array}{c}\% \\
\text { Ceiling }\end{array}$ \\
\hline Overall & $\mathrm{I}, 155$ & $0.36-0.38$ & $0.78-0.84$ & $0.14-0.27$ & $24.4-29.2$ & .96 & 0 & 17.3 \\
\hline Informal & 448 & $0.33-0.50$ & $0.67-0.76$ & $-0.01-0.21$ & $11.4-14.8$ & .93 & 0 & 13.4 \\
\hline Formal & 397 & $0.24-0.33$ & $0.78-0.92$ & $0.32-0.56$ & |3.4-19.1 & .97 & 0 & 17.5 \\
\hline Indian & 101 & $0.11-0.20$ & $0.77-0.93$ & $0.18-0.40$ & $6.7-13.8$ & .97 & 0 & 20.3 \\
\hline White & 107 & $0.21-0.39$ & $0.66-0.86$ & $0.30-0.47$ & $3.3-9.3$ & .94 & 0 & 18.1 \\
\hline Mixed Race & 102 & $0.21-0.33$ & $0.82-0.91$ & $0.12-0.25$ & $8.2-11.7$ & .97 & 0 & 17.9 \\
\hline
\end{tabular}

Note. RSES $=$ Rosenberg Self-Esteem scale.

${ }^{a}$ Range for equivalent item variance. ${ }^{b}$ Range of correlations between items and hypothesized scale, corrected for overlap. ${ }^{c}$ Range of correlations

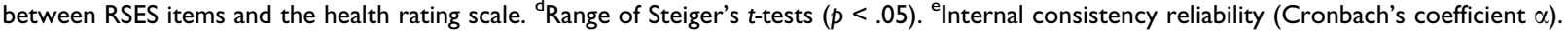


0.98; Indian: ICC 95\% CI: 0.96-0.98; and Mixed Race respondents: ICC 95\% CI: 0.96-0.98), Table 1. Inspection of the last column in the SPSS output (Cronbach's alpha if item deleted) revealed that deleting Q4, Q9 and Q10 would cause alpha to drop from 0.94 to 0.93 for the White group. This did not apply to the other four groups - alpha remained the same irrespective of item deletion.

Floor and ceiling effects. Table 1 also shows floor and ceiling effects overall and for the five groups. The results showed that less than one percent of respondents scored at the floor, but there was substantial variability in the percentage of respondents scoring at the ceiling across sub-groups. Informal settlement residents had a much lower ceiling than the other four groups.

Component structure. Parallel analysis compared the random data eigenvalues with the actual data eigenvalues (O’Connor, 2000). Random data eigenvalues were 1.08 and 1.06. Actual data eigenvalues were 7.29 and 0.60 . The actual data eigenvalue of 7.29 was greater than 1.08 and 0.60 was less than 1.06, retaining one component. In accordance with Schmitt and Allik's (2005) analyses, principal components analysis (PCA) examined the 10 RSES items. One factor was extracted and accounted for $72.9 \%$ of the variance for the overall South African (SA) sample, $61.1 \%$ of the variance for informal settlement residents, $80.2 \%$ of the variance for formal township residents, $66.5 \%$ of the variance for White respondents, $80.2 \%$ of the variance for Indian respondents, and $79.8 \%$ of the variance for Mixed Race respondents (Table 2).

Factor loadings ranged between 0.82 and 0.88 (overall SA), 0.73 and 0.81 (informal settlement), 0.82 and 0.94 (formal township), 0.72 and 0.90 (Whites), 0.81 and 0.95 (Indians), and 0.85 and 0.93 (Mixed Races), fulfilling the criterion of $>0.50$ for factor loadings (Nunnally, 1978), Table 2. All loadings were in excess of 0.71 , indicating that the 10 
Table 2. Factor Structure and Proportion of Variance Explained in the RSES for the Five Groups Compared With Botswana and Zimbabwe.

\begin{tabular}{|c|c|c|c|c|c|c|c|c|c|c|c|c|}
\hline \multirow[b]{2}{*}{ Group } & \multirow[b]{2}{*}{$N$} & \multicolumn{10}{|c|}{ Principal Component Loadings } & \multirow{2}{*}{$\begin{array}{c}\text { Proportion of } \\
\text { Variance Explained }\end{array}$} \\
\hline & & QI & Q2 & Q3 & Q4 & Q5 & Q6 & Q7 & Q8 & Q9 & Q10 & \\
\hline Overall (SA) & 1155 & 0.85 & 0.84 & 0.86 & 0.87 & 0.87 & 0.87 & 0.88 & 0.85 & 0.84 & 0.82 & $72.9 \%$ \\
\hline Informal & 448 & 0.79 & 0.76 & 0.80 & 0.81 & 0.79 & 0.79 & 0.80 & 0.73 & 0.78 & 0.78 & $61.1 \%$ \\
\hline Formal & 397 & 0.89 & 0.88 & 0.91 & 0.91 & 0.93 & 0.94 & 0.92 & 0.90 & 0.82 & 0.84 & $80.2 \%$ \\
\hline Indian & 101 & 0.81 & 0.82 & 0.91 & 0.93 & 0.89 & 0.91 & 0.89 & 0.92 & 0.95 & 0.93 & $66.5 \%$ \\
\hline White & 107 & 0.80 & 0.81 & 0.81 & 0.90 & 0.72 & 0.82 & 0.81 & 0.79 & 0.85 & 0.85 & $80.2 \%$ \\
\hline Mixed race & 102 & 0.85 & 0.90 & 0.87 & 0.87 & 0.89 & 0.93 & 0.91 & 0.92 & 0.92 & 0.88 & $79.8 \%$ \\
\hline Botswana & 213 & 0.55 & 0.64 & 0.64 & 0.41 & 0.66 & 0.52 & 0.63 & 0.29 & 0.50 & 0.51 & $29.8 \%$ \\
\hline Zimbabwe & 193 & 0.65 & 0.60 & 0.56 & 0.55 & 0.55 & 0.58 & 0.57 & 0.29 & 0.62 & 0.64 & $32.4 \%$ \\
\hline
\end{tabular}

Note. RSES = Rosenberg Self-Esteem scale; SA = South Africa. 
Table 3. Mean Scores for the Rosenberg Self-Esteem Scale (RSES), Self-Competence (SC), Self-Liking (SL), Positive (Positive SE), and Negative (Negative SE) Self-Esteem for the Five Groups.

\begin{tabular}{|c|c|c|c|c|c|c|c|c|c|c|c|}
\hline & \multirow[b]{2}{*}{$N$} & \multicolumn{2}{|c|}{ RSES } & \multicolumn{2}{|c|}{ Self-Competence (SC) } & \multicolumn{2}{|c|}{ Self-Liking (SL) } & \multicolumn{2}{|c|}{ Positive SE } & \multicolumn{2}{|c|}{ Negative SE } \\
\hline & & M & $S D$ & M & $S D$ & M & $S D$ & M & $S D$ & M & $S D$ \\
\hline Overall & 1155 & 35.03 & 5.23 & 17.48 & 2.70 & 17.56 & 2.67 & 17.53 & 2.72 & $|7.5|$ & 2.69 \\
\hline Informal & 448 & 32.40 & 5.05 & 16.19 & 2.64 & 16.22 & 2.62 & 16.26 & 2.70 & 16.14 & 2.63 \\
\hline Formal & 397 & 36.30 & 4.88 & 18.04 & 2.60 & 18.27 & 2.38 & 18.07 & 2.62 & 18.24 & 2.37 \\
\hline Indian & 101 & 38.10 & 3.61 & 19.13 & 1.70 & 18.97 & 1.95 & 19.13 & 1.69 & 18.97 & 1.98 \\
\hline White & 107 & 36.86 & 4.33 & 18.36 & 2.19 & $|8.5|$ & 2.32 & 18.45 & 2.08 & 18.42 & 2.45 \\
\hline Mixed race & 102 & 36.70 & 4.56 & 18.41 & 2.25 & 18.28 & 2.39 & 18.42 & 2.29 & 18.27 & 2.39 \\
\hline
\end{tabular}


items represented a pure self-esteem factor for each of the five groups (Tabachnick \& Fidell, 1996, p. 677).

The Kaiser-Meyer-Olkin (KMO) measure of item sampling adequacy was 0.94 (informal settlement) and 0.92 (formal township), in the 'marvelous' category, according to Kaiser (1974). KMO was 0.81 (Whites) and 0.89 (Indians and Mixed Races), in the 'meritorious" category (Kaiser, 1974).

Socio-demographic effects on the RSES, SC and SL

Linear regression analyses evaluated the combined socio-demographic effects (age, gender, schooling, marital status, and employment status) on the RSES, self-competence (SC), and self-liking (SL) for each group. There were no significant relationships between these sociodemographic characteristics and self-esteem, SC, and SL $(p>0.05)$ for formal township residents and Mixed Race residents. For informal settlement residents, schooling $(\beta=0.11, p$ $=0.05)$ and employment status $(\beta=0.11, p=0.03)$ explained $2 \%$ of the variance in the RSES; employment status $(\beta=0.10, p<0.05)$ explained $2 \%$ of the variance in SC and SL, respectively.

For Indian respondents, gender explained $4 \%$ of the variance in the RSES $(\beta=0.26, p$ $<0.05), 4 \%$ of the variance in SC $(\beta=0.26, p<0.01)$, and $3 \%$ of the variance in SL $(\beta=$ $0.25, p<0.05)$. For White respondents, age explained $3 \%$ of the variance in the RSES $(\beta=$ $0.23, p<0.05), 4 \%$ of the variance in SC $(\beta=0.22, p<0.05)$, and $2 \%$ of the variance in SL $(\beta=0.22, p<0.05)$.

Group effects on the RSES, SC, SL, and positive and negative items

Table 3 shows mean scores and standard deviations for overall RSES, self-competence (SC), self-liking (SL), and positively and negatively worded items. All five groups scored above 
the theoretical midpoint (25.00) of the RSES (Schmitt \& Allik, 2005), indicating that generally positive self-evaluations were common. Mean scores for SC and SL were very similar for all five groups. Mean scores on the positively and negatively worded items were similar and significantly inter-related (range: 0.79-0.93) for all five groups, attesting to the internal structure reliability of the RSES (Schmitt \& Allik, 2005). Informal settlement respondents scored significantly lower $(p<0.001)$ than the other four groups on the RSES, its two dimensions SC and SL, and positively and negatively worded items.

\section{Discussion}

The RSES is probably the most widely used measure of global self-esteem, self-competence (SC), and self-liking (SL) in social science research. Although the 53 nation study (Schmitt \& Allik, 2005) addressed many of the concerns that have been raised about the use of the RSES in cross-cultural and cross-racial settings, three issues required further exploration: the lack of formal tests of item validity, generalization of the findings (Sinclair et al., 2010), and the assumption that African cultures are collectivistic (Triandis, 1995).

The current study attempted to address these issues in several ways. First, samples of adults with a wide age range came from five historically distinct areas of Greater Pretoria: one Black formal township and one Black informal settlement, one White, one Indian, and one Mixed Race suburb. Each group had separate psychometric analyses. In addition to the tests conducted by Schmitt and Allik (2005), multi-trait scaling determined item-level psychometric properties. Examination of the effects of socio-demographic characteristics on the RSES, self-competence (SC), and self-liking (SL) contributed an additional analysis. The effects of individualism-collectivism in comparison with Maslow's (1954) hierarchy of needs examined global self-esteem, SC, and SL. For each of the five groups, most scaling assumptions (equivalent item variance, item convergent and discriminant validity, and 
internal consistency reliability) met the criteria. Low variance of responses to the 10 RSES items indicated that each of these five groups responded comparably to all items (Schmitt \& Allik, 2005). For each of the five groups, all 10 corrected item-total correlation coefficients were higher than the criterion, providing evidence for item convergent validity (Stewart, Hays \& Ware, 1988). Steiger's (1980) $t$ tests for dependent correlations showed that the 10 RSES items were better measures of global self-esteem than global health, providing evidence for discriminant validity.

Reliability (internal consistency) coefficients ranged between 0.93 and 0.97 . These reliability coefficients were remarkably similar for the five groups, which satisfied Nunnally's (1978, p. 245-246) standards of reliability (0.70 minimum for research purposes and 0.95 desirable for applied settings), and was considerably higher than those found in other African countries (Schmitt \& Allik, 2005). For example, coefficient alpha was 0.72 in Botswana and 0.75 in Zimbabwe. It would appear that self-esteem, in its global format, is a more cohesive concept to the five South African groups, whereas it is a less cohesive concept in Botswana and Zimbabwe, since responses to the RSES were less consistent (Schmitt \& Allik, 2005).

The results on floor effects for the total South African sample were similar to those reported for a total US sample. However, ceiling effects were slightly higher at $17.3 \%$ in comparison with $16.0 \%$ for the US sample (Sinclair et al., 2010). Ceiling effects ranged between $17.5 \%$ and $18.1 \%$ for Formal Township, White, and Mixed Race respondents suggesting that these three groups perceived global self-esteem as a similar concept. Ceiling effects for the two Black groups were $13.4 \%$ and $17.5 \%$, which is considerably lower than the 22.2\% reported for African-Americans (Sinclair et al., 2010). These findings suggest that the RSES more adequately assesses global self-esteem for Black South Africans than for African-Americans. 
Parallel analysis determined the extraction of one factor. The amount of variance ranged between $61.1 \%$ and $80.2 \%$, considerably higher than that of $29.8 \%$ for Botswana and 32.4\% for Zimbabwe (Schmitt \& Allik, 2005). For the five groups, factor loadings were in excess of 0.71, indicative of a pure self-esteem factor (Tabachnick \& Fidell, 1996). In Botswana, two items (Q4: "I am able to do things as well as others" and Q8: "I wish I could have more respect for myself') did not fulfill Nunnally's (1978) criterion for factor loadings; the latter item was equally problematic in Zimbabwe (Schmitt \& Allik, 2005). According to Schmitt and Allik (2005), the item (I wish I could have more respect for myself) "contains a degree of ambiguity that may cause it to be easily misinterpreted in some cultures" (p. 627). This appears to apply to Botswana and Zimbabwe, since the factor loading was 0.29 on this item.

Conflicting South African findings have emerged on this item, with one study reporting problematic issues for Black, but not White, respondents (Bornman, 1999) and the other study reporting a factor loading of 0.78 for disadvantaged Black respondents (Westaway \& Maluka, 2005). In the current study, factor loadings for this item ranged between 0.75 (informal settlement residents) and 0.92 (Indian and Mixed Race respondents), suggesting that there was no ambiguity for these respondents.

Parallel analysis and one factor model provided evidence on the one-dimensionality of the RSES (Rosenberg, 1965; Westaway \& Maluka, 2005), which contrasts with the duality of positive and negative self-esteem factors reported in previous South African research (Bornman, 1999). Factor loadings and the measure of item sampling adequacy provided support for content and construct validity (Kaiser, 1974; Kim \& Mueller, 1978; Nunnally, 1978; Pullmann \& Allik, 2000; Rosenberg, 1965; Schmitt \& Allik, 2005; Sitzia, 1999; Tabachnick \& Fidell, 1996; Westaway \& Maluka, 2005). 
Linear regression analyses examined the combined effects of age, gender, schooling, marital status, and employment status on the RSES and the self-competence (SC) and selfliking (SL) dimensions. These analyses showed that age, gender and employment status explained less than 5\% of the variance in RSES, SC, and SL. Previous US research has found that global self-esteem increased with age (Orth, Trzesniewski \& Robins, 2010; Sinclair et al., 2010). However, the findings on gender were conflicting, with women having lower selfesteem than men do in young adulthood (Orth, Trzesniewski \& Robins, 2010), and no differences between men and women on self-esteem (Sinclair et al., 2010). In contrast with these findings, global self-esteem, SC, and SL increased with age for White respondents only, and being female led to higher levels of self-esteem, SC, and SL for Indian respondents only. Working led to higher levels of self-esteem, SC, and SL for informal settlement respondents only. This finding was most likely due to the high levels of unemployment among the other four groups.. Although these relationships provided support for the minor role of sociodemographic characteristics in global self-esteem, SC, and SL (Westaway \& Maluka, 2005), it is possible that the samples of White and Indian respondents were not representative of these populations.

All five groups scored above the theoretical midpoint of the RSES, as did respondents from Botswana and Zimbabwe, suggesting that generally positive evaluations appear to be universal (Schmitt \& Allik, 2005). For the five South African groups, the relationships between positively and negatively worded items attested to internal structure reliability, congruence between positive and negative items, and no negative biases in response (Schmitt \& Allik, 2005).

Mean scores for SC and SL were very similar for all five groups, in contrast with the US study (Sinclair et al., 2010), where SC was generally higher than SL. Mean scores on SC were higher than SL in Botswana and Zimbabwe (Schmitt \& Allik, 2005). This finding 
suggested that there was more concordance between SC and SL than was found in the US, Botswana, and Zimbabwe (Schmitt \& Allik, 2005; Sinclair et al., 2010). As Botswana is a collectivistic culture (Schmitt \& Allik, 2005, p. 635), this finding did not provide support for higher SC scores in individualistic cultures (Sinclair et al., 2010; Tafarodi, Lang \& Smith, 1999). These findings challenged the assumption that African cultures are collectivistic than individualistic (Eaton \& Louw, 2000; Triandis, 1995). It would appear that the effects of acculturation, rapid urbanization, and globalization (Naidoo \& Mahabeer, 2006) contribute to the concordance between SC and SL for these five South African groups. For each of the five groups, the relationship between SC and SL suggested that there were two separate, but interrelated, dimensions of global self-esteem (Tafarodi, Lang \& Smith, 1999; Tafarodi \& Milne, 2002; Tafarodi \& Swann, 1995).

There were significant differences between informal settlement respondents and the other four groups on global self-esteem, SC and SL, and the positively and negatively worded items. Informal settlement residents are at a considerable disadvantage in terms of their housing structures and provision of basic services (Westaway \& Maluka, 2005), in comparison with formal township residents, Indians, Whites, and Mixed Races. It is possible that these differences were due to physiological needs taking precedence over higher order needs (Maslow, 1954; Veenhoven, 1996; Westaway, 2006).

\section{Conclusions and Limitations}

The results from the present study demonstrated that the RSES was psychometrically sound and provided support for content and construct validity. It would appear that the RSES, selfcompetence (SC), and self-liking (SL) are particularly useful in measuring self-esteem in this South African setting, since socio-demographic characteristics played a minor role. 
Satisfaction of basic needs seemed to be more important for self-evaluation than individualism-collectivism.

Out of the five comparisons with Botswana and Zimbabwe (Schmitt \& Allik, 2005), the only finding that complemented their findings was that positive evaluation is universal. Contrasting findings may have been due to sampling or more rapid urbanization in South Africa than in these neighboring countries.

Limitations included the sampling strategy for White, Indian and Mixed Race respondents, low levels of education, the high unemployment rate, and sample sizes. This sampling strategy may have accounted for the significant age and gender effects for White respondents and Indian respondents, and the lack of any socio-demographic effects for Mixed Race respondents. As 39\% of the sample had seven or less years of formal schooling and only $20 \%$ were working, this lack of variability limited power in detecting differences. A rule-of-thumb for PCA is 10 persons per item (Nunnally, 1978). The two Black groups easily met this criterion. The White, Indian, and Mixed Race subgroups barely met this criterion. Confirmatory factor analysis (CFA), in addition to PCA, tests the fit of the model (Sinclair, et al., 2010). However, this would have meant that there were 10 indicators (10 RSES items), which were the manifest variables, five covariance matrices, and five mean vectors used for the data in the analysis; a total of 150 model parameters (50 path coefficients, 50 variance estimates, and 50 mean/intercept estimates). As there were only 107 White, 101 Indian, and 102 Mixed Race respondents, fit indices would most likely not indicate a strong fit of the model to the data (Paquet \& Kline, 2009). Therefore, much larger samples of Whites, Indians, and Mixed Races are required in order to conduct CFA. 


\section{References}

Alexopoulos, E.C., \& Geitona, M. (2010). Self-rated health: Inequalities and potential determinants. International Journal of Environmental Research and Public Health, 6, 2456-2469.

Arias, E., \& de Vos, S. (1996). Using housing items to indicate socioeconomic status: Latin America. Social Indicators Research, 38, 53-80.

Baumeister, R.F., \& Tice, D.M. (1988). Metatraits. Journal of Personality, 56, 571-598.

Bornman, E. (1999). Self-image and ethnic identification in South Africa. Journal of Social Psychology, 139, 411-425.

Carmines, E.G., \& Zeller, R.A. (1979). Reliability and validity assessment. Beverley Hills, CA: Sage.

Cronbach, L.J. (1970). Essentials of psychological testing (3rd ed.). New York: Harper \& Row, 160-161.

Diener, E., \& Diener, M. (1995). Cross-cultural correlates of life satisfaction and self-esteem. Journal of Personality and Social Psychology, 68, 653-663.

Eaton, L., \& Louw, J. (2000). Culture and self in South Africa: Individualism-collectivism predictions. Journal of Social Psychology, 140, 210-217.

Francis, L.J., \& James, D.J. (1996). The relationship between Rosenberg's construct of selfesteem and Eysenck's two-dimensional model of personality. Personality and Individual Differences, 21, 483-488.

George, D., \& Mallery, P. (2003). SPSS for windows step by step: A simple guide and reference 11.0 Update (4th ed.). Boston: Allyn \& Bacon, 231.

Gilmore, A., McKee, M., \& Rose, M. (2002). Determinants of and inequalities in selfperceived health in Ukraine. Social Science and Medicine, 55, 2177-2188. 
Greenberger, E., Chen, C., Dmitrieva, J., \& Farruggia, S.P. (2003). Item-wording and the dimensionality of the Rosenberg Self-Esteem Scale: Do they matter? Personality and Individual Differences, 35, 1241-1254.

Hatcher, J., \& Hall, L.A. (2009). Psychometric properties of the Rosenberg Self-Esteem Scale in African American single mothers. Issues in Mental Health Nursing, 30, 7077.

Hofstede, G. (2001). Culture consequences. Comparing values, behaviours, institutions, and organizations across nations (2nd ed.). Thousand Oaks, CA: Sage.

Idler, E., \& Benjamin, Y. (1997). Self-rated health and mortality: A review of twenty-seven community studies. Journal of Health and Social Behavior, 38, 21-27.

Kaiser, H.F. (1974). An index of factorial simplicity. Psychometrika, 39, 31-36.

Kim, J., \& Mueller, CW. (1978). Factor analysis: Statistical methods and practical issues. Newbury Park: Sage.

Lehohla, P. (2006). Stats in brief, 2006. Pretoria: Statistics South Africa.

Maslow, A. (1954). Motivation and personality. New York: Harper \& Row.

Meurer, L.N., Layde, P.M., \& Guse, C.E. (2001). Self-rated health status: A new vital sign for primary care? Wisconsin Medical Journal, 100, 35-39.

Naidoo, J.C., \& Mahabeer, M. (2006). Acculturation and integration patterns among Indian and African university students in South Africa: Implications for ethno-gender relations in the "Rainbow" nation. Psychology and Developing Societies, 18, 115-132.

Nunnally, J.C. (1978). Psychometric theory (2nd ed.). New York: McGraw-Hill.

O'Connor, B.P. (2000). SPSS and SAS programs for determining the number of components using parallel analysis and Velicer's MAP test. Behavior Research Methods, Instruments, \& Computers, 32, 396-402. 
Orth, U., Trzesniewski, K.H., \& Robins, R.W. (2010). Self-esteem development from young adulthood to old age: A cohort-sequential longitudinal study. Journal of Personality and Social Psychology, 98, 645-658.

Paquet, S.L., \& Kline, T.J.B. (2009). Uncovering the psychometric properties of scales measuring Individualist and Collectivist orientations. International Journal of Testing, 9, 260-270.

Pullmann, H., \& Allik, J. (2000). The Rosenberg Self-Esteem Scale: Its dimensionality, stability and personality correlates in Estonian. Personality and Individual Differences, 28, 701-715.

Rosenberg, M. (1965). Society and the adolescent child. Princeton, NJ: Princeton University Press.

Schmitt, D.P., \& Allik, J. (2005). Simultaneous administration of the Rosenberg Self-Esteem Scale in 53 nations: exploring the universal and culture-specific features of global self-esteem. Journal of Personality and Social Psychology, 89, 623-642.

Sinclair, S.J., Blais, M.A., Gansler, D.A., Sandberg, E., Bistis, K., \& LoCicero, A. (2010). Psychometric properties of the Rosenberg Self-Esteem Scale: Overall and across demographic groups living within the United States. Evaluation \& the Health Professions, 33, 56-80.

Sitzia, J. (1999). How valid and reliable are patient satisfaction data? An analysis of 195 studies. International Journal for Quality in Health Care, 11, 319-328.

Steiger, J.H. (1980). Tests for comparing elements of a correlation matrix. Psychological Bulletin, 87, 245-251.

Stewart, A.L., Hays, R.D., \& Ware, J.E. (1988). The MOS Short-form General Health Survey: Reliability and validity in a patient population. Medical Care, 26, 724-735. 
Tabachnick, B.G., \& Fidel, L.S. (1996). Using multivariate statistics (3rd ed.). New York: HarperCollins.

Tafarodi, R.W., Lang, J.M., \& Smith, A.J. (1999). Self-esteem and the cultural trade-off: Evidence for the role of individualism-collectivism. Journal of Cross-Cultural Psychology, 30, 620-64.

Tafarodi, R.W., \& Milne, A.B. (2002). Decomposing global self-esteem. Journal of Personality, 70, 443-483.

Tafarodi, R.W., \& Swann, W.B. (1995). Self-liking and self-competence as dimensions of global self-esteem: Initial validation of a measure. Journal of Personality Assessment, $65,322-342$.

Triandis, H.C. (1995). Individualism and collectivism. Boulder, CO: Westview.

Veenhoven, R. (1996). Developments in satisfaction-research. Social Indicators Research, $37,1-46$.

Westaway, M.S. (2006). A longitudinal investigation of satisfaction with personal and environmental quality of life in an informal South African housing settlement: Doornkop, Soweto. Habitat International, 30, 175-189.

Westaway, M.S. (2007). Life and neighborhood satisfaction of Black and White residents in a middle-class suburb of Johannesburg. Psychological Reports, 100, 489-494.

Westaway, M.S., \& Maluka, C.S. (2005). Are life satisfaction and self-esteem distinct constructs? A black South African perspective. Psychological Reports, 97, 567-575. 